\title{
On the computation of Hermite-Humbert constants for real quadratic number fields
}

\author{
par Michael E. POHST et MARCUS WAGNER
}

\begin{abstract}
RÉSUMÉ. Nous présentons des algorithmes pour le calcul des formes de Humbert binaires extrémales sur les corps quadratiques réels. Grâce à ces algorithmes, nous sommes capables de calculer les formes de Humbert extrémales pour les corps de nombres $\mathbb{Q}(\sqrt{13})$ et $\mathbb{Q}(\sqrt{17})$. Enfin nous calculons la constante d'HermiteHumbert pour le corps de nombres $\mathbb{Q}(\sqrt{13})$.

ABSTRACT. We present algorithms for the computation of extreme binary Humbert forms in real quadratic number fields. With these algorithms we are able to compute extreme Humbert forms for the number fields $\mathbb{Q}(\sqrt{13})$ and $\mathbb{Q}(\sqrt{17})$. Finally we compute the Hermite-Humbert constant for the number field $\mathbb{Q}(\sqrt{13})$.
\end{abstract}

\section{Introduction}

A new invariant of number fields, called the Hermite-Humbert constant, was introduced by M.I. Icaza in 1997 (see [Ica]). This constant is an analogue to the Hermite constant for $\mathbb{Q}$. In $[\mathrm{H}]$ Humbert describes a generalization of the reduction theory for quadratic forms over $\mathbb{Z}$ where he considers totally positive forms, called Humbert forms, with integral entries from a given number field $K$. He deduces an analogous reduction theory and the existence of the Hermite-Humbert constant.

In this work we compute extreme binary Humbert forms for the number fields $\mathbb{Q}(\sqrt{13})$ and $\mathbb{Q}(\sqrt{17})$ and the Hermite-Humbert constant $\gamma_{K, 2}$ for $\mathbb{Q}(\sqrt{13})$. In [BCIO] it is shown that finding Hermite-Humbert constants of real quadratic number fields is tantamount to looking for extreme Humbert forms. Their existence is proved in [Ica]. Following the precedence of Voronoï a characterization of extreme Humbert forms is given in [C] by introducing two properties of extreme Humbert forms, namely perfection and eutacticity. 


\section{The theoretical background}

Let $\mathcal{P}$ denote the set of positive real binary Humbert forms, i.e. $S=$ $\left(S_{1}, S_{2}\right) \in \mathcal{P}$ with positive definite $2 \times 2$ real matrices $S_{1}$ and $S_{2}$. If $K$ denotes a real quadratic number field and $\mathcal{O}_{K}$ its maximal order, we denote with $S[x]$ the product $x^{t} S_{1} x \cdot x^{\prime t} S_{2} x^{\prime}$ for any $x \in \mathcal{O}_{K}^{2}$, where $x^{\prime}$ denotes the conjugate vector of $x$ and we let $\operatorname{det} S$ be the product of the determinants $\operatorname{det} S_{1}$ and $\operatorname{det} S_{2}$. In the same way $U^{\prime}$ denotes the matrix $U \in K^{2 \times 2}$ with all entries conjugated. Let

$$
m(S):=\min \left\{S[x]: 0 \neq x \in \mathcal{O}_{K}^{2}\right\}
$$

denote the minimum of a given Humbert form $S$, then

$$
M(S):=\left\{[x] \in \mathcal{O}_{K}^{2} \mid S[x]=m(S)\right\}
$$

denotes a set of equivalence classes

$$
[x]:=\left\{y \in \mathcal{O}_{K}^{2} \mid y=\epsilon x, \epsilon \in \mathcal{O}_{K}^{\times}\right\}
$$

where the elements of $M(S)$, respectively their representatives, are called minimal vectors of $S$. To avoid superfluous notation we denote the elements $[x]$ of $M(S)$ only with $x$. If a tuple $U:=\left(U_{1}, U_{2}\right) \in \mathrm{GL}\left(2, \mathcal{O}_{K}\right)^{2}$ is given we can make unimodular transformations from a Humbert form $S$ to another denoted by

$$
S[U]:=\left(S\left[U_{1}\right], S\left[U_{2}\right]\right):=\left(U_{1}^{t} S_{1} U_{1}, U_{2}^{t} S_{2} U_{2}\right) .
$$

By scaling we mean multiplication of a given Humbert form $S=\left(S_{1}, S_{2}\right)$ with an element $\lambda=\left(\lambda_{1}, \lambda_{2}\right) \in\left(\mathbb{R}^{>0}\right)^{2}$ to obtain another Humbert form

$$
\lambda S:=\left(\lambda_{1} S_{1}, \lambda_{2} S_{2}\right) .
$$

We note that for a given Humbert form $S$ the set $M(S)$ is finite which is shown in [Ica]. The following theorem is due to Humbert $([\mathrm{H}])$. He proved the existence of Hermite-Humbert constants:

Theorem 1. Let $K$ be a real quadratic number field. Then for any $S \in \mathcal{P}$ there is a constant $C \in \mathbb{R}^{>0}$ such that

$$
S[x] \leq C \sqrt{\operatorname{det} S} \quad \forall x \in M(S) .
$$

The best known upper bounds for $C$ are given in [Co2] and [Ica]. We use the estimate $C \leq \frac{1}{2}\left|d_{K}\right|$ from [Co2]. With theorem 1 we are able to define a map from $\mathcal{P}$ to $\mathbb{R}^{>0}$ in the following way.

Definition \& Proposition 2. Let $S \in \mathcal{P}$ and

$$
\gamma_{K}: \mathcal{P} \longrightarrow \mathbb{R}^{>0}, \quad \gamma_{K}(S)=\frac{m(S)}{\sqrt{\operatorname{det} S}},
$$

then $\gamma_{K}$ is invariant under unimodular transformations and scaling. 
Now we are able to define the Hermite-Humbert constant as

$$
\gamma_{K, 2}=\sup _{S \in \mathcal{P}} \gamma_{K}(S)
$$

Any Humbert form $S$ for which equality holds in (2) is called critical. The existence of such forms is shown in [Ica]. The value $\gamma_{K}(S)$ of a critical Humbert form $S$ is a global maximum of $\gamma_{K}$. Forms for which $\gamma_{K}$ achieves a local maximum are called extreme. For characterizing extreme forms we introduce two properties (see [C]): perfection and eutacticity. If $S=$ $\left(S_{1}, S_{2}\right) \in \mathcal{P}$ and $x \in M(S)$, then

$$
\left(\frac{x x^{t}}{S_{1}[x]}, \frac{x^{\prime} x^{\prime t}}{S_{2}\left[x^{\prime}\right]}\right)
$$

is a semi-positive definite Humbert form. The set of such forms of a given $S \in \mathcal{P}$ will be denoted with $X_{S}$. Now perfection means

$$
\operatorname{dim} \sum_{X \in X_{S}} \mathbb{R} X=5
$$

and eutacticity means that there is a representation

$$
S^{-1}=\left(S_{1}^{-1}, S_{2}^{-1}\right)=\sum_{X \in X_{S}} \rho_{X} X
$$

with $\rho_{X} \in \mathbb{R}^{>0}$ for all $X \in X_{S}$. The following theorem of [C] characterizes extreme forms:

Theorem 3. A Humbert form is extreme if and only if it is eutactic and perfect.

So we get the following corollary ([BCIO]):

Corollary 4. An extreme Humbert form of a real quadratic number field has at least 5 minimal vectors.

Now we need the definition of equivalence for two Humbert forms:

Definition 5. Two Humbert forms $S$ and $T$ are called equivalent, if there is a tupel $U:=\left(U_{1}, U_{2}\right) \in \mathrm{GL}\left(2, \mathcal{O}_{K}\right)^{2}$ with $U_{2}=U_{1}^{\prime}$ and

$$
T=S[U]
$$

or if there is $\lambda=\left(\lambda_{1}, \lambda_{2}\right) \in\left(\mathbb{R}^{>0}\right)^{2}$ such that $S=\lambda T$.

In $[C]$ it is shown that there are only a finite number of extreme forms up to equivalence. This suggests we should look for a suitable set of representatives for extreme Humbert forms. With the next lemmata we are able to determine such a set and a finite set $M$ which contains all minimal vectors for each element of this set. To reduce the amount of necessary computations we introduce the following definition: 
Definition 6. Two elements $x, y \in \mathcal{O}_{K}^{2}$ are called a unimodular pair if they are $a \mathcal{O}_{K}$-basis of $\mathcal{O}_{K}^{2}$, i.e. $\mathcal{O}_{K}^{2}=\mathcal{O}_{K} x \oplus \mathcal{O}_{K} y$.

With $\epsilon_{0}>1$ we denote the fundamental unit and with $h_{K}$ the class number of a real quadratic number field $K$. The following lemma is proved in [BCIO]:

Lemma 7. Let $h_{K}=1$. If any extreme Humbert form has a unimodular pair of minimal vectors then it is equivalent to a form

$$
S=\left(\left(\begin{array}{cc}
1 & b_{1} \\
b_{1} & c
\end{array}\right),\left(\begin{array}{cc}
1 & b_{2} \\
b_{2} & c^{-1}
\end{array}\right)\right)
$$

with $\epsilon_{0}^{-1} \leq c<\epsilon_{0}$. The standard basis vectors $e_{1}$ and $e_{2}$ are contained in the set $M(S)$ and for any other minimal vector $x=\left(x_{1}, x_{2}\right)^{t} \in \mathcal{O}_{k}^{2}$ we have

and

$$
\left|\mathrm{N}_{K / \mathbb{Q}}\left(x_{1}\right)\right| \leq \gamma_{K, 2}, \quad\left|\mathrm{~N}_{K / \mathbb{Q}}\left(x_{2}\right)\right| \leq \gamma_{K, 2}
$$

$$
\left|x_{2}^{(1)}\right|<\frac{\sqrt{\epsilon_{0}} \gamma_{K, 2}}{\left|x_{1}^{(2)}\right|}, \quad\left|x_{2}^{(2)}\right|<\frac{\sqrt{\epsilon_{0}} \gamma_{K, 2}}{\left|x_{1}^{(1)}\right|} .
$$

We denote the obtained set of such representatives with $\mathfrak{S}$. In order to restrict the finite set $M$ of minimal vectors for each element of $\mathfrak{S}$ we can make use of the following lemma (see [BCIO]):

Lemma 8. (1) Let $S=\left(S_{1}, S_{2}\right) \in \mathcal{P}$ with

$$
S_{i}=\left(\begin{array}{ll}
a_{i} & b_{i} \\
b_{i} & c_{i}
\end{array}\right)
$$

$$
\begin{aligned}
& \text { for } i=1,2 \text { and } u=\left(u_{1}, u_{2}\right)^{t} \in \mathcal{O}_{K}^{2} \text {. Then } \\
& \begin{aligned}
\left|\mathrm{N}_{K / \mathbb{Q}}\left(u_{1}\right)\right| & \leq \sqrt{\frac{c_{1} c_{2} S[u]}{m(S)^{2}}} \gamma_{K, 2}, \\
\left|\mathrm{~N}_{K / \mathbb{Q}}\left(u_{2}\right)\right| & \leq \sqrt{\frac{a_{1} a_{2} S[u]}{m(S)^{2}}} \gamma_{K, 2}, \\
\left|u_{1}^{(1)} u_{2}^{(2)}\right| & \leq \sqrt{\frac{a_{2} c_{1} S[u]}{m(S)^{2}}} \gamma_{K, 2}, \\
\left|u_{1}^{(2)} u_{2}^{(1)}\right| & \leq \sqrt{\frac{a_{1} c_{2} S[u]}{m(S)^{2}}} \gamma_{K, 2} .
\end{aligned}
\end{aligned}
$$

(2) Let $u=\left(u_{1}, u_{2}\right)^{t}, v=\left(v_{1}, v_{2}\right)^{t} \in \mathcal{O}_{K}^{2}$ be minimal vectors of $S$ with $v \notin \mathcal{O}_{K}^{\times} u$ and

$$
U=\left(\begin{array}{ll}
u_{1} & u_{2} \\
v_{1} & v_{2}
\end{array}\right)
$$


then

$$
\left|\mathrm{N}_{K / \mathbb{Q}}(\operatorname{det} U)\right| \leq \gamma_{K, 2} .
$$

By corollary 4 we know that every extreme Humbert form has at least 5 minimal vectors. Now we are able to compute all possible extreme forms as follows if we assume that every Humbert form has a unimodular pair of minimal vectors:

(1) determine the finite set $M$ of all possible minimal vectors for each element of $\mathfrak{S}$.

(2) for any 3 -set $T=\left\{u_{1}, u_{2}, u_{3}\right\} \subseteq M \backslash\left\{e_{1}, e_{2}\right\}$ we have to solve polynomial equations $S\left[u_{i}\right]=1$ in the unknowns of $S \in \mathfrak{S}$ where

$$
S=\left(\left(\begin{array}{cc}
1 & b_{1} \\
b_{1} & c
\end{array}\right),\left(\begin{array}{cc}
1 & b_{2} \\
b_{2} & c^{-1}
\end{array}\right)\right)
$$

and $i=1,2,3$.

(3) finally we have to test whether the obtained Humbert forms are eutactic and perfect.

If we have two perfect Humbert forms $S$ and $T$ we need an algorithm which decides whether $T$ and $S$ are equivalent. We consider two 3-sets $W=\left\{w_{1}, w_{2}, w_{3}\right\} \subseteq M(T)$ and $V=\left\{v_{1}, v_{2}, v_{3}\right\} \subseteq M(S)$ of minimal vectors. If $S$ and $T$ are unimodular equivalent then there is a matrix $U \in \mathrm{GL}\left(2, \mathcal{O}_{K}\right)$ with

$$
U w_{i}=\epsilon_{i} v_{i}
$$

for $i=1,2,3$ and suitable units $\epsilon_{i} \in \mathcal{O}_{K}^{\times}$. Let $S$ and $T$ be two perfect Humbert forms. Then $S$ and $T$ are equivalent if and only if there exists a matrix $U \in \mathrm{GL}\left(2, \mathcal{O}_{K}\right)$ with $U \cdot M(T)=M(S)$.

\section{The algorithms}

In this section $K$ always denotes a real quadratic number field with $h_{K}=1$ and $\epsilon_{0}>1$ the fundamental unit of the maximal order $\mathcal{O}_{K}$.

We want to develop an algorithm for computing extreme Humbert forms. The main algorithm splits into several subalgorithms. The first subalgorithm for computing the set $M$ and all 3-sets is algorithm 10. After computing all 3 -sets of $M$ we have to compute real solutions of the polynomial equations obtained by all triples of $M$ in order to construct Humbert forms. Then we compute minimal vectors with algorithm 14 and, if necessary, eutactic coefficients. Finally we test the obtained Humbert forms for equivalence by algorithm 11 . 
Algorithm 9 (Main Algorithm).

Input: $\quad$ The maximal order $\mathcal{O}_{K}$ of a real quadratic number field $K$

Output: A set of all eutactic and perfect Humbert forms of $K$ up to equivalence having a unimodular pair of minimal vectors

- $T \leftarrow$ All 3-sets of M

- $H_{1} \leftarrow$ set of Humbert forms obtained by the real solutions of the polynomial equations

- $H_{2} \leftarrow\left\{S=\left(S_{1}, S_{2}\right) \in H_{1} \mid m(S)=1\right\}$

- $H_{3} \leftarrow\left\{\left(h_{1}, h_{2}\right) \mid h_{2} \in H_{2}, h_{1}=\right.$ set of minimal vectors of $h_{2}$ and $\left.\left|h_{1}\right|>4\right\}$

- $H_{4} \leftarrow\left\{h=\left(h_{1}, h_{2}\right) \in H_{3} \mid h_{2}\right.$ is perfect and eutactic $\}$

- $H_{5} \leftarrow$ set of non-equivalent Humbert forms

- Return $\mathrm{H}_{5}$

3.1. Computing of all 3 -sets of $M$. In this section we describe the algorithm to compute all suitable 3 -sets of $M$. We make use of the results of lemma 7 and lemma 8 . Note that we assume every Humbert form has got a unimodular pair of minimal vectors.

Algorithm 10 (3-sets of $M$ ).

Input: An integral basis $1, \omega$ of the maximal order $\mathcal{O}_{K}$ of a real quadratic number field $K$, the fundamental unit $\epsilon_{0}$

Output: All suitable 3-sets of $M$

- $M \leftarrow \emptyset, \quad X, Y \leftarrow\left\{[\alpha] \mid \alpha \in \mathcal{O}_{K}\right.$ with $\left.0<\left|\mathrm{N}_{K / \mathbb{Q}}(\alpha)\right| \leq \frac{d_{K}}{2}\right\}$

- For all $x \in X$ do

For all $y \in Y$ do

if $\exists k \in \mathbb{Z}$ with $\left|\left(y \epsilon_{0}^{k}\right)^{(1)}\right|<\frac{\sqrt{\epsilon_{0}} d_{K}}{x^{(2)}}$ and $\left|\left(y \epsilon_{0}^{k}\right)^{(2)}\right|<\frac{\sqrt{\epsilon_{0}} d_{K}}{x^{(1)}}$ then

$$
M \leftarrow M \cup\left\{\left(x, y \epsilon_{0}^{k}\right)^{t}\right\}
$$

- $T \leftarrow$ set of all 3 -sets of $M$

- $S \leftarrow \emptyset$

- For all $A=\left\{\alpha_{i} \mid \alpha_{i} \in \mathcal{O}_{K}^{2}, i=1,2,3\right\} \in T$ do

$\Psi \leftarrow\left\{\left(\alpha_{i}, \alpha_{j}\right) \in \mathrm{GL}(2, K) \mid i, j \in\{1,2,3\}\right\}$

If $\Psi=\emptyset$ or for any $X \in \Psi$ there holds $\left|\mathrm{N}_{K / \mathbb{Q}}(\operatorname{det} X)\right|>\frac{d_{K}}{2}$ then

$$
S \leftarrow S \cup\{A\}
$$

Else 


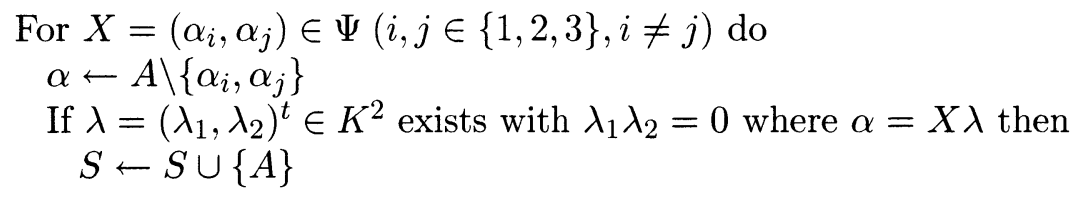

- Return $T \backslash S$

3.2. Unimodular equivalent Humbert forms. Now we describe an algorithm which decides whether two perfect Humbert forms $S$ and $T$ are unimodular equivalent. Let $A=\left\{a_{1}, a_{2}, a_{3}\right\} \subseteq M(S)$ and let us assume there holds

$$
\lambda_{2} a_{2}=a_{1}
$$

with $\lambda_{2} \in K$. If $\lambda_{2}=\frac{\lambda_{21}}{\lambda_{22}}\left(\lambda_{22}, \lambda_{21} \in \mathcal{O}_{K}, \lambda_{22} \neq 0\right)$ with $\operatorname{gcd}\left(\lambda_{22}, \lambda_{21}\right)=1$ then $a_{1}=\lambda_{21} \mu$ for some $\mu \in \mathcal{O}_{K}^{2}$. Now we get

$$
S\left[a_{1}\right]=\mathrm{N}_{K / \mathbb{Q}}\left(\lambda_{21}\right)^{2} S[\mu]
$$

and we obtain $\left|\mathrm{N}_{K / \mathbb{Q}}\left(\lambda_{21}\right)\right|=1=\left|\mathrm{N}_{K / \mathbb{Q}}\left(\lambda_{22}\right)\right|$. Together this means

$$
\lambda_{2} a_{2}=a_{1} \Rightarrow\left[a_{1}\right]=\left[a_{2}\right] .
$$

The same holds if we change the role of the $a_{i}(i=1,2,3)$. If $A=$ $\left\{a_{1}, a_{2}, a_{3}\right\} \subseteq M(S)$ and $B=\left\{b_{1}, b_{2}, b_{3}\right\} \subseteq M(T)$ then let us assume withous loss of generality the first two elements of $A$ and $B$ are $K$-linear independent. Now we have a representation of the third element of each set in terms of the first two. It is easy to see that if a matrix $U \in \mathrm{GL}\left(2, \mathcal{O}_{K}\right)$ exists fulfilling (4) then for the matrices $X:=\left(a_{1}, a_{2}\right)$ and $Y:=\left(b_{1}, b_{2}\right)$ there holds $Y X^{-1} \in \mathrm{GL}\left(2, \mathcal{O}_{K}\right)$. If we assume the existence of a $U \in \mathrm{GL}\left(2, \mathcal{O}_{K}\right)$ fulfilling (4) we have

$$
U\left(a_{1}, a_{2}, a_{3}\right)=\left(\epsilon_{1} b_{1}, \epsilon_{2} b_{2}, b_{3}\right) \quad\left(\epsilon_{1}, \epsilon_{2} \in \mathcal{O}_{K}^{\times}\right)
$$

and with $a_{3}=X \lambda$ and $b_{3}=Y \mu$ we get $\lambda=\left(\lambda_{1}, \lambda_{2}\right)^{t}, \mu=\left(\mu_{1}, \mu_{2}\right)^{t} \in K^{2}$ with $\lambda_{1}, \lambda_{2}, \mu_{1}, \mu_{2} \neq 0$ because of (6). Now we obtain

$$
\epsilon_{1}=\frac{\mu_{1}}{\lambda_{1}} \text { and } \epsilon_{2}=\frac{\mu_{2}}{\lambda_{2}} \text {. }
$$

Finally we have to test whether $\epsilon_{1}$ and $\epsilon_{2}$ are units in $\mathcal{O}_{K}$ and

$$
\left(\left(\epsilon_{1} b_{1}, \epsilon_{2} b_{2}\right) X^{-1}\right) M(S)=M(T) .
$$

Algorithm 11 (Equivalence of Humbert Tuples).

Input: $\quad$ The sets $M(S)$ and $M(T)$ of two perfect Humbert forms $S$ and $T$ of a real quadratic number field $K$

Output: $\quad U \in \mathrm{GL}\left(2, \mathcal{O}_{K}\right)$ with $U \cdot M(S)=M(T)$ if $S$ and $T$ are unimodular equivalent, false otherwise 
- $S \leftarrow \emptyset$

- For all $A=\left\{a_{i} \mid a_{i} \in \mathcal{O}_{K}^{2}, i=1,2,3\right\} \subseteq M(S)$

For all $B=\left\{b_{i} \mid b_{i} \in \mathcal{O}_{K}^{2}, i=1,2,3\right\} \subseteq M(T)$ do

$$
\begin{aligned}
& \Psi_{1} \leftarrow\left\{\left(a_{i}, a_{j}\right) \in \mathrm{GL}(2, K) \mid i, j \in\{1,2,3\}\right\} \\
& \Psi_{2} \leftarrow\left\{\left(b_{i}, b_{j}\right) \in \mathrm{GL}(2, K) \mid i, j \in\{1,2,3\}\right\} \\
& \text { For } X=\left(a_{i}, a_{j}\right) \in \Psi_{1} \text { and } Y=\left(b_{k}, b_{l}\right) \in \Psi_{2} \text { do } \\
& \text { If }\left|\mathrm{N}_{K / \mathbb{Q}}\left(\operatorname{det} Y X^{-1}\right)\right|=1 \text { then } \\
& \quad a \leftarrow A \backslash\left\{a_{i}, a_{j}\right\} \\
& \quad b \leftarrow B \backslash\left\{b_{k}, b_{l}\right\} \\
& \quad \lambda \leftarrow\left(\lambda_{1}, \lambda_{2}\right)^{t} \in K^{2} \text { with } \lambda_{1} \lambda_{2} \neq 0 \text { where } a=X \lambda \\
& \mu \leftarrow\left(\mu_{1}, \mu_{2}\right)^{t} \in K^{2} \text { with } \mu_{1} \mu_{2} \neq 0 \text { where } b=Y \mu \\
& \epsilon_{1} \leftarrow \frac{\mu_{1}}{\lambda_{1}} \\
& \epsilon_{2} \\
& \text { If } \epsilon_{1}, \epsilon_{2} \in \epsilon_{2} \in \mathcal{O}_{K}^{\times} \\
& \quad \text { If }\left(\epsilon_{1} b_{k}, \epsilon_{2} b_{l}\right) X^{-1} \cdot M(S)=M(T) \text { then } \\
& \quad \operatorname{Return}\left(\left(\epsilon_{1} b_{k}, \epsilon_{2} b_{l}\right) X^{-1}\right)
\end{aligned}
$$

- Return false

3.3. Computing minimal vectors. Next we want to compute minimal vectors of a given $S \in \mathcal{P}$. To do this we need a constructive proof for the finiteness of minimal vectors of a given Humbert form. ( We will need certain quantities from this proof for the following algorithm which computes minimal vectors).

Lemma 12. Let $S=\left(S_{1}, S_{2}\right) \in \mathcal{P}, C \in \mathbb{R}^{>0}$ and $K$ be a real quadratic number field. Then the set

$$
\left\{x \in \mathcal{O}_{K}^{2} \mid S_{1}[x]+S_{2}\left[x^{\prime}\right] \leq C\right\}
$$

is finite.

Proof. For all $T \in \mathbb{R}^{2 \times 2}$ we denote with $\underline{\|T\|}$ the value

$$
\min _{x \neq 0} \frac{\|T x\|_{2}}{\|x\|_{2}} \quad \forall x \in \mathbb{R}^{2} .
$$

If $T$ is a regular matrix then we have

$$
\underline{\|T\|}=\min _{x \neq 0} \frac{\|T x\|_{2}}{\|x\|_{2}}=\min _{y \neq 0} \frac{\|y\|_{2}}{\left\|T^{-1} y\right\|_{2}}=\left\|T^{-1}\right\|_{2}^{-1}
$$

with $y=T x$. If we consider the Cholesky decompositions for $S_{i}=R_{i}^{t} R_{i}$ with $R_{i} \in \mathrm{GL}(2, \mathbb{R})(i=1,2)$, we get

$$
S_{1}[x]=\left\|R_{1} x\right\|_{2}^{2} \geq \underline{\left\|R_{1}\right\|^{2}}\|x\|_{2}^{2}=\left\|R_{1}^{-1}\right\|_{2}^{-2}\|x\|_{2}^{2}
$$


and the same holds for $S_{2}\left[x^{\prime}\right]$. For the computation of $\left\|R_{i}^{-1}\right\|_{2}$ we need the well known estimate

$$
\left\|R_{i}\right\|_{2} \leq\left(\left\|R_{i}\right\|_{\infty}\left\|R_{i}\right\|_{1}\right)^{\frac{1}{2}} \quad(i=1,2)
$$

and now we get with (8)

$$
S_{1}[x] \geq\left\|R_{1}^{-1}\right\|_{2}^{-2}\|x\|_{2}^{2} \geq\left(\left\|R_{1}^{-1}\right\|_{\infty}\left\|R_{1}^{-1}\right\|_{1}\right)^{-1}\|x\|_{2}^{2}
$$

where the same holds again for $S_{2}\left[x^{\prime}\right]$. If $m:=\min _{i=1,2}\left(\left\|R_{i}^{-1}\right\|_{\infty}\left\|R_{i}^{-1}\right\|_{1}\right)^{-1}$ we get with $x=(a, b)^{t} \in \mathcal{O}_{K}^{2}$

$$
m\left(a^{2}+a^{\prime 2}+b^{2}+b^{\prime 2}\right)=m\left(\|x\|_{2}^{2}+\left\|x^{\prime}\right\|_{2}^{2}\right) \leq S_{1}[x]+S_{2}\left[x^{\prime}\right] \leq C
$$

and further

$$
a^{2}+a^{\prime 2}=\left(a, a^{\prime}\right)\left(a, a^{\prime}\right)^{t}=\underbrace{\left(\left(\begin{array}{cc}
1 & 1 \\
\omega & \omega^{\prime}
\end{array}\right)\left(\begin{array}{cc}
1 & \omega \\
1 & \omega^{\prime}
\end{array}\right)\right)}_{:=A}\left[\left(a_{1}, a_{2}\right)^{t}\right]
$$

with $a=a_{1}+a_{2} \omega$ for an integral basis $\{1, \omega\}$ of $\mathcal{O}_{K}$ and $a_{1}, a_{2} \in \mathbb{Z}$. We know the matrix $A$ is a positive definite form in $\mathbb{Z}$ because the trace of integral elements of a given maximal order are rational integral elements. With (9) and (10) we obtain for any element $z=\left(x_{1}+x_{2} \omega, y_{2}+y_{2} \omega\right)^{t}\left(x_{1}, x_{2}, y_{1}, y_{2} \in\right.$ $\mathbb{Z}$ ) of the set in $(7)$ the condition

$$
A\left[\left(x_{1}, x_{2}\right)^{t}\right] \leq \frac{C}{m} \text { and } A\left[\left(y_{1}, y_{2}\right)^{t}\right] \leq \frac{C}{m}
$$

and the set of solutions of these inequalities is finite and so the set in (7) is finite, too.

The next lemma motivates lemma 12 .

Lemma 13. Let $S=\left(S_{1}, S_{2}\right) \in \mathcal{P}$. Then there exists a constant $\alpha \in$ $\mathbb{R}^{>0}$ such that suitable representants of the elements of the set $M(S)$ are contained in the set

$$
\left\{x \in \mathcal{O}_{K}^{2} \mid S_{1}[x]+S_{2}\left[x^{\prime}\right] \leq \alpha\right\}
$$

Proof. This is an easy consequence of the fact that there is only a finite number of integers in a given number field with bounded absolute values of their conjugates.

With the last two lemmata we are able to compute minimal vectors of a given $S \in \mathcal{P}$. 
Algorithm 14 (Minimal Vectors).

Input: A Humbert form $S=\left(S_{1}, S_{2}\right) \in \mathcal{P}$, the maximal order $\mathcal{O}_{K}$ with integral basis $\{1, \omega\}, \alpha$ and $m$ as in lemma 12 and lemma 13

Output: The set $J \subseteq \mathcal{O}_{K}^{2}$ of all minimal vectors of $S$

- $I, J \leftarrow \emptyset$

$S_{i} \leftarrow C S_{i} \quad(i=1,2)$ where $S_{i}=\left(\begin{array}{ll}a_{i} & b_{i} \\ b_{i} & c_{i}\end{array}\right)$ and $C=\left(\max \left\{a_{1}, a_{2}\right\}\right)^{-1}$

$\epsilon_{0} \leftarrow$ the fundamental unit $\epsilon$ of $\mathcal{O}_{K}$ with $\epsilon>1$

$A \leftarrow\left(\begin{array}{cc}\operatorname{Tr}_{K / \mathbb{Q}}(1) & \operatorname{Tr}_{K / \mathbb{Q}}(\omega) \\ \operatorname{Tr}_{K / \mathbb{Q}}(\omega) & \operatorname{Tr}_{K / \mathbb{Q}}\left(\omega^{2}\right)\end{array}\right)$

$B \leftarrow\left(\begin{array}{cc}1 & \ln \left|\epsilon_{0}^{2}\right| \\ 1 & \ln \left|\epsilon_{0}^{\prime 2}\right|\end{array}\right)$

- $L \leftarrow\left\{\left(x_{1}, x_{2}\right)^{t} \in \mathcal{O}_{K}^{2} \mid x_{i}=\left(x_{i 1}+x_{i 2} \omega\right), x_{i 1}, x_{i 2} \in \mathbb{Z}\right.$ and

$$
\left.A\left[\left(x_{i 1}, x_{i 2}\right)^{t}\right] \leq \frac{\alpha}{m},(i=1,2)\right\}
$$

- $\mu \leftarrow \min _{x \in L} S[x]$

For $x \in L$ do

If $S[x]=\mu$ then $I \leftarrow I \cup\{x\}$

- For $x \in I$ do

$$
\begin{aligned}
& k \leftarrow n \in \mathbb{Z} \text { with } 0 \leq \lambda_{2}-n<1 \text { where } \\
& \lambda=\left(\lambda_{1}, \lambda_{2}\right)^{t} \in \mathbb{R}^{2} \text { with } B \lambda=\left(\begin{array}{c}
\ln S_{1}[x] \\
\ln S_{2}\left[x^{\prime}\right]
\end{array}\right)
\end{aligned}
$$

$$
J \leftarrow J \cup\left\{x \epsilon^{-k}\right\}
$$

- Return $J$

3.4. Computing eutactic coefficients. For computing eutactic coefficients we use well known combinatorical algorithms. There exist classical algorithms to compute barycentric coordinates based on linear programming.

\section{Examples}

Now we have the theoretical background and the algorithms for computing extreme Humbert forms. The algorithms are implemented in KASH/KANT (see [Pohst et al]). To continue we need the following lemma: 
Lemma 15. Let $S \in \mathcal{P}$ and $v_{i}=\left(\alpha_{i}, \beta_{i}\right)^{t} \in M(S)$ for $1 \leq i \leq s:=|M(S)|$ and for $1 \leq i \neq j \leq s$ let $v_{i j}$ be the determinants of the corresponding pairs:

$$
v_{i j}=\operatorname{det}\left(\begin{array}{cc}
\alpha_{i} & \alpha_{j} \\
\beta_{i} & \beta_{j}
\end{array}\right) \text {. }
$$

Then, for a fixed prime ideal $\mathfrak{p}$, with corresponding exponential valuation $\nu_{\mathfrak{p}}$, we have: If $\{i, j, k\} \subseteq\{1, \ldots, s\}$ is ordered so that

$$
\nu_{\mathfrak{p}}\left(v_{i j}\right) \geq \max \left(\nu_{\mathfrak{p}}\left(v_{i k}\right), \nu_{\mathfrak{p}}\left(v_{j k}\right)\right)
$$

then

$$
\nu_{\mathfrak{p}}\left(v_{i j}\right) \geq \nu_{\mathfrak{p}}\left(v_{i k}\right)=\nu_{\mathfrak{p}}\left(v_{j k}\right) .
$$

Proof. For a proof see $[\mathrm{BCIO}]$

Now we can use our algorithms to compute the Hermite-Humbert constant for the case $K=\mathbb{Q}(\sqrt{13})$. We need the following lemma:

Lemma 16. If $K=\mathbb{Q}(\sqrt{13})$ and $\gamma_{K, 2} \leq \frac{d_{K}}{2}=6.5$ then any Humbert form $S$ with more than 4 minimal vectors has got a unimodular pair of minimal vectors.

Proof. Let us assume there is no unimodular pair in $M(S)$. With the notations of lemma 15 we obtain $\left|\mathrm{N}_{K / \mathbb{Q}}\left(\operatorname{det} v_{i j}\right)\right| \leq \gamma_{K, 2}$ for each $v_{i j}$ by lemma 8 and because of $|M(S)| \geq 5$ we have at least $\left(\begin{array}{l}5 \\ 2\end{array}\right)=10$ of the $v_{i j}$. Because of $h_{K}=1$ we can always assume $e_{1} \in M(S)$. With $v_{5}:=e_{1}$ we have $v_{5 i}=\beta_{i}$. Because of $\gamma_{K, 2} \leq \frac{d_{K}}{2}=6.5$ and the assumption above we obtain $\nu_{\mathfrak{p}_{k}}\left(v_{i j}\right)=1$ for at least $k=1,2$ or 3 where $\mathfrak{p}_{1}=(2)$ and $\mathfrak{p}_{2} \mathfrak{p}_{3}=(3)$. At first we consider without lost of generality the situation $\nu_{\mathfrak{p}_{1}}\left(v_{15}\right)=1=\nu_{\mathfrak{p}_{1}}\left(v_{25}\right)$ and $v_{\mathfrak{p}_{2}}\left(\nu_{35}\right)=1$. That means $\nu_{\mathfrak{p}_{3}}\left(v_{13}\right)=1=\nu_{\mathfrak{p}_{3}}\left(v_{23}\right)$ because otherwise we would get a contradiction like $\nu_{\mathfrak{p}_{1}}\left(v_{35}\right)=1, \nu_{\mathfrak{p}_{2}}\left(v_{15}\right)=1$ or $\nu_{\mathfrak{p}_{2}}\left(v_{25}\right)=1$. So we obtain by lemma 15

$$
1=\nu_{\mathfrak{p}_{3}}\left(v_{23}\right) \geq \nu_{\mathfrak{p}_{3}}\left(v_{12}\right)=\underbrace{\nu_{\mathfrak{p}_{3}}\left(v_{13}\right)}_{=1}
$$

and then $\nu_{\mathfrak{p}_{3}}\left(v_{12}\right)=1=\nu_{\mathfrak{p}_{1}}\left(v_{12}\right)$, a contradiction.

Lastly there is the case where all $\beta_{i}$ are divisible by a single prime ideal. Without loss of generality let $\nu_{\mathfrak{p}_{1}}\left(\beta_{i}\right)=1$ for $i \in\{1, \ldots, 4\}$. By assumption there is $\nu_{\mathfrak{p}_{1}}\left(v_{12}\right)=1=\nu_{\mathfrak{p}_{1}}\left(\alpha_{1} \beta_{2}-\alpha_{2} \beta_{1}\right)=\nu_{\mathfrak{p}_{1}}(\gamma)+\nu_{\mathfrak{p}_{1}}\left(\alpha_{1} \epsilon_{2}-\alpha_{2} \epsilon_{1}\right)$ with $\beta_{i}=\gamma \epsilon_{i}$ where $\nu_{\mathfrak{p}_{1}}(\gamma)=1, \epsilon_{1}, \epsilon_{2} \in \mathcal{O}_{K}$ are $\mathfrak{p}_{1}$-units and $\gamma \in \mathcal{O}_{K}$. Because of $\left|\mathcal{O}_{K} / \mathfrak{p}_{1}\right|=4$ and $s \geq 5$ we can assume by changing $v_{1}$ and $v_{2}$ if necessary that $\nu_{\mathfrak{p}_{1}}\left(\alpha_{1}-\alpha_{2}\right)>0$, but then $\nu_{\mathfrak{p}_{1}}\left(\alpha_{1} \epsilon_{2}-\alpha_{2} \epsilon_{1}\right)>0$, a contradiction. 
By algorithm 10 we obtain about 4000 triples. Because of lemma 16 we know each extreme Humbert form must have a unimodular pair of minimal vectors. We found 770 Humbert forms with more than 4 minimal vectors. By algorithm 11 we obtain 3 Humbert forms with more than four minimal vectors up to unimodular equivalence. Their minimal vectors are listed in the following table:

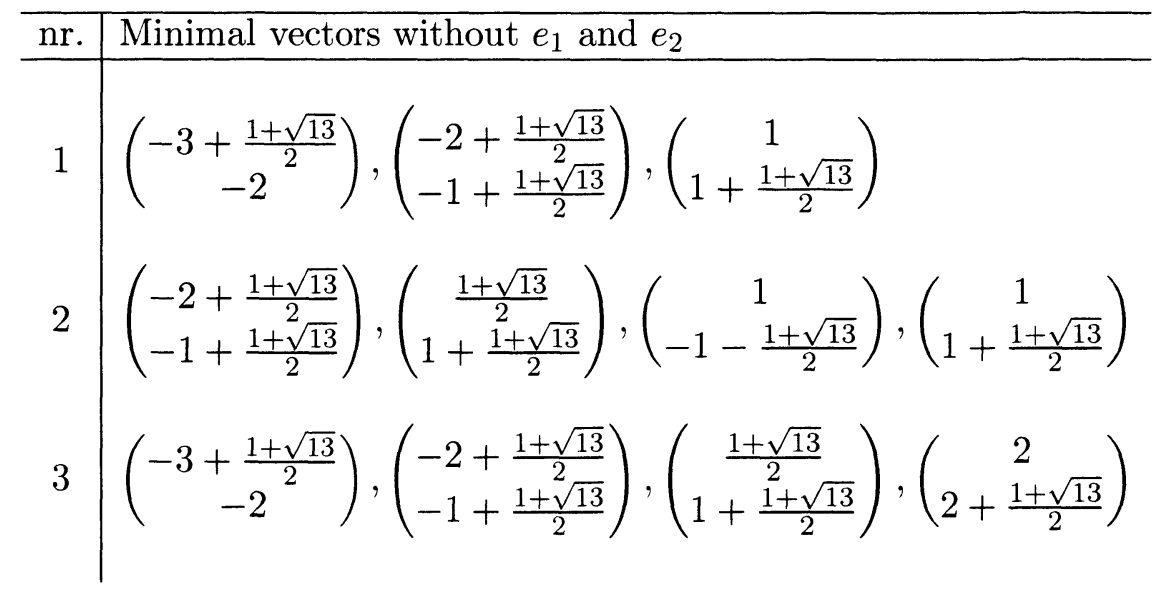

Remark 17. The Humbert form $T$ resulting from the minimal vectors of the first row in the table above is an extreme form. But the value of $\gamma_{K}(T)$ is less then the value of the Humbert form $S$ below. The Humbert form resulting from the minimal vectors of the third row in the table above has perfection of dimension 3.

We obtain a critical Humbert form $S=\left(S_{1}, S_{2}\right)$ with

$$
\begin{aligned}
& S_{1}=\left(\begin{array}{cc}
1 & \frac{-8+\sqrt{13}+7 \sqrt{7}-2 \sqrt{91}}{18} \\
\frac{-8+\sqrt{13}+7 \sqrt{7}-2 \sqrt{91}}{18} & \frac{7-2 \sqrt{13}-14 \sqrt{7}+4 \sqrt{91}}{9}
\end{array}\right) \\
& S_{2}=\left(\begin{array}{cc}
1 & \frac{-8-\sqrt{13}-7 \sqrt{7}-2 \sqrt{91}}{18} \\
\frac{-8-\sqrt{13}-7 \sqrt{7}-2 \sqrt{91}}{18} & \frac{7+2 \sqrt{13}+14 \sqrt{7}+4 \sqrt{91}}{9}
\end{array}\right) .
\end{aligned}
$$

with $m(S)=1$. For its eutactic coefficients we compute

$$
\rho_{1}=\rho_{2}=\frac{9+\sqrt{91}}{28}, \quad \rho_{3}=\rho_{6}=\frac{3(11-\sqrt{91})}{70}, \quad \rho_{4}=\rho_{5}=\frac{29+\sqrt{91}}{140}
$$

and for its minimal vectors we get $M(S) \backslash\left\{x_{3}:=e_{2}, x_{6}:=e_{1}\right\}=$

$$
\left\{x_{1}=\left(\begin{array}{c}
\frac{-3+\sqrt{13}}{2} \\
\frac{-1+\sqrt{13}}{2}
\end{array}\right), x_{2}=\left(\begin{array}{c}
\frac{1+\sqrt{13}}{2} \\
\frac{3+\sqrt{13}}{2}
\end{array}\right), x_{4}=\left(\begin{array}{c}
1 \\
-\frac{3+\sqrt{13}}{2}
\end{array}\right), x_{5}=\left(\begin{array}{c}
1 \\
\frac{3+\sqrt{13}}{2}
\end{array}\right)\right\} \text {. }
$$


After verifying

$$
S^{-1}=\sum_{i=1}^{6} \rho_{i}\left(\frac{x_{i} x_{i}^{t}}{S_{1}[x]}, \frac{x_{i}^{\prime} x_{i}^{\prime t}}{S_{2}\left[x^{\prime}\right]}\right)
$$

and $\operatorname{dim} \sum_{X \in X_{S}} \mathbb{R} X$ is equal to 5 , we get

$$
\gamma_{K}(S)=\gamma_{K, 2}=\sqrt{\frac{1476+144 \sqrt{91}}{175}}=4.0353243 \ldots
$$

because of $S$ being critical.

In the case $\mathbb{Q}(\sqrt{17})$ we were not able to show that each extreme Humbert form has a unimodular pair of minimal vectors. But we can use our algorithms to compute extreme forms with pairs of unimodular minimal vectors. With Algorithm 10 we compute about 80000 triples. Now we solve polynomial equations to obtain Humbert forms and compute minimal vectors and if necessary eutactic coefficients. We give an example with the triple

$$
\left\{\left(\begin{array}{c}
\frac{-5+\sqrt{17}}{2} \\
1
\end{array}\right),\left(\begin{array}{c}
\frac{-3+\sqrt{17}}{2} \\
-1
\end{array}\right),\left(\begin{array}{c}
1 \\
-1
\end{array}\right)\right\} \text {. }
$$

If we write a Humbert form $S$ as

$$
S=\left(\left(\begin{array}{cc}
1 & b_{1} \\
b_{1} & c
\end{array}\right),\left(\begin{array}{cc}
1 & b_{2} \\
b_{2} & c^{-1}
\end{array}\right)\right)
$$

we obtain for every minimal vector $x=\left(x_{1}, x_{2}\right)^{t} \in \mathcal{O}_{K}^{2}$ polynomial equations in $c, b_{1}$ and $b_{2}$

$$
S[x]-1=0 \Leftrightarrow\left(x_{1}^{2}+2 b_{1} x_{1} x_{2}+c x_{2}^{2}\right)\left(c x_{1}^{\prime 2}+2 c b_{2} x_{1}^{\prime} x_{2}^{\prime}+x_{2}^{\prime 2}\right)-c=0 .
$$

We use resultants of polynomials for eliminating $b_{1}$ and $b_{2}$ to obtain a polynomial in $c$ like

$$
\begin{aligned}
f(c)= & c^{6}-\frac{3}{2} c^{5}+\frac{-1360+330 \sqrt{17}}{8} c^{4}+\frac{2970-720 \sqrt{17}}{4} c^{3} \\
& +\frac{1360-330 \sqrt{17}}{8} c^{2}+\frac{-13062+3168 \sqrt{17}}{4} c+\frac{4354-1056 \sqrt{17}}{2} .
\end{aligned}
$$

Now we obtain by factoring

$$
\begin{aligned}
& f(c)=\left(c^{2}+\frac{16-4 \sqrt{17}}{2} c+\frac{16 \sqrt{17}-66}{2}\right) \\
& \cdot\left(c^{2}+\frac{1-\sqrt{17}}{4} c+\frac{-9+\sqrt{17}}{8}\right) \cdot\left(c^{2}+\frac{9 \sqrt{17}-39}{4}+\frac{161-39 \sqrt{17}}{8}\right)
\end{aligned}
$$


and for $g(c):=c^{2}+(8-2 \sqrt{17}) c+8 \sqrt{17}-33$ we get

$$
c_{1,2}=(\sqrt{17}-4) \pm \sqrt{2(33-8 \sqrt{17})} .
$$

Because of $(-4+\sqrt{17})^{2}=33-8 \sqrt{17}$ the solutions $c_{1,2}$ are in the field $L:=\mathbb{Q}(\sqrt{17}, \sqrt{2})$ and we obtain by $c>0$

$$
c=\sqrt{17}-4+\sqrt{2} \sqrt{17}-4 \sqrt{2} .
$$

Then we substitute $c$ into the polynomial

$$
\begin{aligned}
& \left(-(128+32 \sqrt{17}) c^{2}-16 c\right) b_{1}^{2} \\
& +\left((264+64 \sqrt{17}) c^{3}+(64+20 \sqrt{17}) c^{2}+8 c\right) b_{1}-(132+32 \sqrt{17}) c^{4} \\
& -(68+16 \sqrt{17}) c^{3}-(12+2 \sqrt{17}) c^{2}+(32-8 \sqrt{17}) c
\end{aligned}
$$

to obtain

$$
b_{1}^{2}+\left(\frac{13}{4}-\sqrt{17}+\sqrt{34}-\frac{17}{4} \sqrt{2}\right) b_{1}+\left(\frac{\sqrt{17}}{2}-\frac{15}{8}+\frac{17 \sqrt{2}}{8}-\frac{\sqrt{34}}{2}\right)=0
$$

which leads to $b_{1}=\frac{1}{2}$. In a last step we substitute the solutions into the polynomial

$$
\begin{aligned}
& \left(8 c b_{1}-(5+\sqrt{17}) c^{2}+(-10+2 \sqrt{17}) c\right) b_{2} \\
+ & (-(10+2 \sqrt{17}) c+(-5+\sqrt{17})) b_{1}+\frac{21+5 \sqrt{17}}{2} c^{2}+4 c+\frac{21-5 \sqrt{17}}{2}
\end{aligned}
$$

and obtain

$$
(7 \sqrt{17}-29) b_{2}+\frac{29-7 \sqrt{17}}{2}=0
$$

with $b_{2}=\frac{1}{2}$. Now we get the following Humbert form $S=\left(S_{1}, S_{2}\right)=$

$$
\left(\left(\begin{array}{ll}
1 & \frac{1}{2} \\
\frac{1}{2} & -4+\sqrt{17}^{\frac{1}{2}}-4 \sqrt{2}+\sqrt{34}
\end{array}\right),\left(\begin{array}{cc}
1 & \frac{1}{2} \\
\frac{1}{2} & -4-\sqrt{17}+4 \sqrt{2}+\sqrt{34}
\end{array}\right)\right)
$$

with $m(S)=1$. For the eutactic coefficients we get

$$
\rho_{1}=\rho_{2}=\rho_{3}=\rho_{4}=\frac{8+\sqrt{34}}{30}, \quad \rho_{5}=\frac{14-2 \sqrt{34}}{15}
$$

and for the minimal vectors we compute $M(S) \backslash\left\{x_{5}:=e_{1}, x_{3}:=e_{2}\right\}=$

$$
\left\{x_{1}:=\left(\begin{array}{c}
\frac{-5+\sqrt{17}}{2} \\
1
\end{array}\right), x_{2}:=\left(\begin{array}{c}
-3+\sqrt{17} \\
2 \\
-1
\end{array}\right), x_{4}:=\left(\begin{array}{c}
1 \\
-1
\end{array}\right)\right\} \text {. }
$$


Together we obtain

$$
S^{-1}=\sum_{i=1}^{5} \rho_{i}\left(\frac{x_{i} x_{i}^{t}}{S_{1}[x]}, \frac{x_{i}^{\prime} x_{i}^{\prime t}}{S_{2}\left[x^{\prime}\right]}\right)
$$

and $\operatorname{dim} \sum_{X \in X_{S}} \mathbb{R} X$ is equal to 5 . Finally we compute

$$
\gamma_{K}(S)=\sqrt{\frac{784+128 \sqrt{34}}{225}}=2.607989300 \ldots
$$

as a local maximum of $\gamma_{K}$.

In the same way we obtain an extreme Humbert form $S=\left(S_{1}, S_{2}\right)$ with

$$
\begin{aligned}
& S_{1}=\left(\begin{array}{cc}
1 & \frac{3+5 \sqrt{17}-3 \sqrt{5}-\sqrt{85}}{16} \\
\frac{3+5 \sqrt{17}-3 \sqrt{5}-\sqrt{85}}{16} & \frac{3-\sqrt{5}}{2}
\end{array}\right), \\
& S_{2}=\left(\begin{array}{cc}
1 & \frac{3-5 \sqrt{17}+3 \sqrt{5}-\sqrt{85}}{16} \\
\frac{3-5 \sqrt{17}+3 \sqrt{5}-\sqrt{85}}{16} & \frac{3+\sqrt{5}}{2}
\end{array}\right)
\end{aligned}
$$

and $m(S)=1$. For the eutactic coefficients we get

$$
\begin{array}{lll}
\rho_{1}=\frac{12}{45}, & \rho_{2}=\frac{44-2 \sqrt{85}}{45}, & \rho_{3}=\frac{11+\sqrt{85}}{45}, \\
\rho_{4}=\frac{12}{45}, & \rho_{5}=\frac{11+\sqrt{85}}{45} &
\end{array}
$$

and for the minimal vectors we compute $M(S) \backslash\left\{x_{5}:=e_{1}, x_{3}:=e_{2}\right\}=$

$$
\left\{x_{1}:=\left(\begin{array}{c}
\frac{3-\sqrt{17}}{2} \\
1
\end{array}\right), x_{2}:=\left(\begin{array}{c}
\frac{5-\sqrt{17}}{2} \\
\frac{3-\sqrt{17}}{2}
\end{array}\right), x_{4}:=\left(\begin{array}{c}
1 \\
\frac{-3-\sqrt{17}}{2}
\end{array}\right)\right\} .
$$

Together we obtain

$$
S^{-1}=\sum_{i=1}^{5} \rho_{i}\left(\frac{x_{i} x_{i}^{t}}{S_{1}[x]}, \frac{x_{i}^{\prime} x_{i}^{\prime t}}{S_{2}\left[x^{\prime}\right]}\right)
$$

and $\operatorname{dim} \sum_{X \in X_{S}} \mathbb{R} X$ is equal to 5 . Finally We compute

$$
\gamma_{K}(S)=\sqrt{\frac{1408+128 \sqrt{85}}{405}}=2.527919014 \ldots
$$

Acknowledgement: We thank the referee for various insightful comments. 


\section{References}

[BCIO] R. Baeza. R. Coulangeon. M.I. Icaza. M. O' Ryan, Hermite's constant for quadratic number fields. Experimental Mathematics 10 (2001), 543-551.

[C] R. Coulangeon, Voronoï theory over algebraic number fields. Monographies de l'Enseignement Mathématique 37 (2001), 147-162.

[Co1] H. CoHn, A numerical survey of the floors of various Hilbert fundamental domains. Math. Comp. 19 (1965), 594-605.

[Co2] H. Conn, On the shape of the fundamental domain of the Hilbert modular group. Proc. Symp. Pure Math. 8 (1965), 190-202.

[H] P. HUMBerT, Théorie de la réduction des formes quadratique définies positives dans un corps algébrique K fini. Comment. Math. Helv. 12 (1940), 263-306.

[Ica] M.I. ICAZA, Hermite constant and extreme forms for algebraic number fields. J. London Math. Soc. 55 (1997), 11-22.

[Pohst et al] M.E. POHST ET AL, The computer algebra system KASH/KANT, TU Berlin 2000, http://www. math.tu-berlin.de/ kant/

Michael E. PoHST

Technische Universität Berlin

Fakultät II

Institut für Mathematik MA 8-1

Str. d. 17. Juni 136

D-10623 Berlin, Germany

E-mail : pohst@math.tu-berlin.de

Marcus WAGNER

Technische Universität Berlin

Fakultät II

Institut für Mathematik MA 8-1

Str. d. 17. Juni 136

D-10623 Berlin, Germany

E-mail : wagner@math.tu-berlin.de 